\title{
Functional Performance Testing in Athletes with
}

\section{Functional Ankle Instability}

\author{
Nidhi Sharma*, MSPT; Archna Sharma, MSPT; Jaspal Singh Sandhu, MS, FAIS, FASM
}

\author{
Authors' Affiliation: \\ Faculty of Department of Sports \\ Medicine and Physiotherapy, \\ Guru Nanak Dev University, \\ Amritsar, Punjab, India \\ * Corresponding Author; \\ Address: Faculty of Sports \\ Medicine and Physiotherapy, \\ Guru Nanak Dev University, \\ Amritsar, Punjab, India \\ E-mail: nids4rehab@gmail.com
}

Received: Apr 05, 2011

Accepted: Aug 05, 2011

Key Words: Joint Instability; Ankle; Athletic Performance; Functional Performance

\begin{abstract}
Purpose: To determine if functional performance deficits are present in athletes with functional ankle instability (FAI) compared to healthy athletes using various functional performance tests.

Methods: Sixty two athletes (mean age-21.7 \pm 1.8 years; height-168.2 $\pm 9.1 \mathrm{~cm}$; weight-63.8 $\pm 11.0 \mathrm{~kg}$ ) participated in this case control study. Athletes were divided into two groups: athletes with FAI (FAI group, $n=31$ ) and healthy athletes (Non-FAI group, $n=31$ ). The FAI group was further divided into two subgroups: FAI with giving way (FAI-GW), FAI with no giving way (FAI-NGW). Functional performance was assessed with the single-limb hopping test, figure-of-8 hop test, side-hop test, single-limb hurdle test, square hop test and single hop test.
\end{abstract}

Results: Significant differences $(P<0.05)$ were observed for all the functional performance tests (FPTs) except the single hop test between FAI and Non-FAI groups; between FAI-GW, FAI-NGW and Non-FAI groups. Additionally, the involved limb performed significantly worse $(P<0.05)$ than the contra-lateral uninvolved limb of the FAI-GW group for the above-mentioned FPTs.

Conclusion: Significant functional performance deficits were observed in the FAI group in all tests except single hop test with greater deficits observed in the FAI-GW group. Hence, these tests can be used to determine the presence of FAI. However no deficits were identified for the test involving sagittal plane functional activities suggesting that this test can not be used as a criterion to discriminate individuals with FAI. It was further ascertained that functional performance was not affected by limb dominance.

Asian Journal of Sports Medicine, Volume 2 (Number 4), December 2011, Pages: 249-258

\section{INTRODUCTION}

Ankle sprain injuries are the most common injury sustained during sporting activities ${ }^{[1]}$. Three-quarters of ankle injuries involve the lateral ligament complex

${ }^{[1]}$. Eighty percent of acute ankle sprains make a full recovery with conservative management, while $20 \%$ of acute ankle sprains develop mechanical or functional instability, resulting in chronic ankle instability ${ }^{[1]}$. The functional ankle instability (FAI) is characterized by a feeling of "giving way" ${ }^{[2-8]}$ and a "disabling loss of reliable static and dynamic support of a joint" ${ }^{[9]}$. The mechanism of recurrent ankle injury is not thought to be different than that of initial acute ankle sprains; however adverse changes that occur after primary injury are believed to predispose individuals to recurrent sprains ${ }^{[10]}$. Two theories of the cause have traditionally been postulated: mechanical instability 
and functional instability ${ }^{[11]}$. Mechanical instability of the ankle complex occurs as a result of anatomic changes after initial ankle sprain, which lead to insufficiencies that predispose the ankle to further episodes of instability ${ }^{[11]}$. These changes include pathologic laxity, impaired arthrokinematics, synovial changes, and the development of degenerative joint disease, which may occur in combination or isolation [11]. The concept of functional instability was first described by Freeman et al ${ }^{[2]}$, who attributed impaired balance in individuals with lateral ankle sprains to damaged articular mechanoreceptors in the lateral ankle ligaments, which resulted in proprioceptive deficits. Furthermore, functional insufficiencies among individuals with either acute ankle sprains or recurrent ankle sprain have been demonstrated by quantifying deficits in ankle proprioception, cutaneous sensation, nerve-conduction velocity, neuromuscular response times, postural control, and strength ${ }^{[11]}$.

Functional performance tests (dynamic full weight bearing tests) are useful predictors of lower extremity performance ${ }^{[12]}$. The functional performance tests (FPTs) include the single legged hop for distance tests, various climbing and walk tests, among others ${ }^{[12-14]}$. By differentially stressing the joints in sagittal and frontal planes as well as combined rotational stress in the transverse plane, these FPTs can be used as a measure to quantify an athlete's level of physical performance by simulating muscular and joint stresses as encountered during actual sporting activities ${ }^{[15,16]}$. Exploiting various FPTs, some studies have found significant difference in performance between FAI group and non-FAI group ${ }^{[5,14,17,18]}$. Functional deficits in participants with ankle instability were found in the FPTs that included movements in the frontal and transverse plane, whereas the tests that solely moved in the sagittal plane did not result in performance deficits [5,19].

However, some studies elicited no functional deficits in participants with FAI ${ }^{[5,19,20]}$. The reason for these discrepancies is unclear, although one potential reason could be the variation in the FPTs chosen to elicit functional performance deficits ${ }^{[5,14,17-20]}$. FPTs such as shuttle run ${ }^{[5,20]}$, single-limb hurdle test ${ }^{[15]}, 6$ meter crossover hop test ${ }^{[18]}$ and agility hop test ${ }^{[19]}$ have been used. This variation could have resulted in the variability of results and may explain the literature's lack of clarity.

Due to the conflicting results of various studies, there is dearth of scientific evidence regarding the exact nature of this condition. Understanding the nature of this condition is necessary to identify the extent of performance deficits related to it and also to set a criterion for return to play for the athletes. Therefore, the aim of this study was to determine if functional performance deficits are present in athletes with FAI during six unilateral hopping tests, including singlelimb hopping test, single-limb hurdle test, figure-of-8 hop test, side-hop test, square hop test and single hop test when compared to their contra-lateral uninvolved ankle and healthy athletes.

Study hypothesis: functional performance deficits are present in athletes with functional ankle instability.

\section{METHODS AND SUBJECTS}

\section{Participants:}

Sixty two athletes (52 males and 10 females) participated in this case control (matched-subject design) study. Participants were matched according to: height, mass, age, type of sport played, level of participation and lower limb dominance. The participants were equally divided into two groups on the basis of presence or absence of FAI: 31athletes with ankle instability in the FAI group (mean age: $21.7 \pm 1.9$ years; mean height: $170.9 \pm 9.3 \mathrm{~cm}$; mean weight: $63.6 \pm 10.9 \mathrm{~kg}$ ) and 31 healthy athletes in the non-FAI group (age: $21.6 \pm 1.8$ years; height: $170.9 \pm 9.1$ $\mathrm{cm}$; weight: $63.9 \pm 11.2 \mathrm{~kg}$ ). Athletes were included in the FAI group on the basis of scores in the Ankle Instability Instrument ${ }^{[5]}$ (day-to-day reliability of this index ranges from 0.86 to 0.70 (standard error of the mean, $\mathrm{SEM}=0.18 \& 0.37$, respectively) ${ }^{[6]}$ (Appendix1). Participants were excluded from the FAI group if they had recent history of injury in the contra-lateral limb, a history of recent injury to the involved lower limb besides the ankle, and/or had symptoms of an acute ankle sprain ${ }^{[18]}$. Participants were excluded from both the groups if they had history of lower extremity 
fracture, lower limb surgery, injury to knee or hip or low back injury ${ }^{[18]}$. An informed consent was taken from all athletes prior to testing. The study was approved by the Institutional Ethical Committee.

\section{Procedure:}

Pre experimental protocol: Each participant visited the laboratory before the start of the study. Before testing, each test was demonstrated along with verbal instructions. To familiarize themselves with the experimental protocol the participants then performed three trials each of the 6 functional performance tests, the single-limb hopping test ${ }^{[14,21]}$, figure-of-8 hop test ${ }^{[5,18]}$, side-hop test ${ }^{[5,18]}$, single-limb hurdle test ${ }^{[14]}$, square hop test ${ }^{[18]}$ and single hop test ${ }^{[5,19]}$. Time taken to complete each test was recorded using a hand-held stop-watch to the nearest 0.01 seconds ${ }^{[5]}$. For the single hop test, the distance was recorded from the position of the toes on the starting line to the end of the jump to the nearest $0.01 \mathrm{~m}{ }^{[5]}$.The limb dominance was determined for each participant by asking them to kick a ball. One minute rest was given after each reading for each test to decrease the chances of athletes becoming fatigued ${ }^{[14]}$.

Instructions to the participants: The athletes were asked to refrain from any strenuous activity for 24 hours before the testing.

Experimental protocol: On the same day following a five minute warm up (on static bicycle), each participant performed each test procedure thrice with maximum effort $\&$ the mean of these 3 readings was recorded as the final score for each individual test. One minute rest was given after each reading for each test and also between successive tests to decrease the chances of participants becoming fatigued ${ }^{[14]}$. If the participant hit the cone during testing (single limb hopping test), touched the contra-lateral foot down and/or hopped out of sequence or out of the designated square, the trial was discarded and repeated again for each test ${ }^{[14]}$.

Single-limb hopping test ${ }^{[14,21]}$ : The single-limb hopping test course was reproduced using the measurements and picture from Chambers et al ${ }^{[21]}$. The course was constructed of hardwood and consisted of eight 13 -in $\times 13$-in $(33.02-\mathrm{cm} \times 33.02-\mathrm{cm})$ squares positioned in two rows of 4 . In each row, the first and last squares were level, and the middle squares were sloped. In 1 row, the 2 middle squares had a $15^{\circ}$ lateral slope; in the other row, 1 square had a $15^{\circ}$ incline, and 1 square had a $15^{\circ}$ decline. Participants were instructed to hop as fast as they could through the course. Trial reliability of the single-limb hopping test was high with an intra-class correlation coefficient (ICC) of 0.93 (SEM, 0.18 second) ${ }^{[14]}$.

Figure-of-8 hop test ${ }^{[5,18]}$ : For the figure-of-8 hop test, a 5-m course outlined by cones was used. Each participant was instructed to hop on 1 limb, twice around the course, as fast as possible. Reliability for this test was excellent, with an ICC of 0.95 (SEM, 1.66 seconds) ${ }^{[18]}$.

Side-hop test ${ }^{[5,18]}$ : For the side-hop test, all participants were instructed to hop on 1 limb laterally over a $30-\mathrm{cm}$ distance. One repetition constituted hopping laterally $30 \mathrm{~cm}$ and back to the starting location. Each participant completed 10 repetitions and was instructed to do so as quickly as possible. Trial reliability was good for this test, with an ICC of 0.84 (SEM, 2.10 seconds) ${ }^{[18]}$.

Single-limb hurdle test ${ }^{[14]}$ : This test has been modified form the study conducted by Buchanan et al ${ }^{[14]}$. The single-limb hurdle test course consisted of 10 squares taped on a concrete floor with 3 small hurdles placed in standard positions on the course. The hurdles were approximately $15 \mathrm{~cm}$ high in the original article, but were reduced to $10 \mathrm{~cm}$ for this study. The hurdles were created from cones connected with athletic tape. The course was adjusted according to the limb tested to ensure that each participant performed 2 lateral jumps and 1 medial jump. Trial reliability for this test was high, with an ICC of 0.93 (SEM, 0.18 second) ${ }^{[14]}$. Square hop test ${ }^{[18]}$ : The square hop consists of a $40 \times$ $40-\mathrm{cm}$ square marked on the floor with tape. Starting outside of the square, participants were instructed to hop in and out of the square as fast as possible for 5 repetitions. One repetition constituted hopping in and out of the tape outline completely around the square back to the starting point. With the right limb, participants hopped in a clockwise direction and with the left limb, they hopped in a counterclockwise direction. Trial reliability for this test was good, with an ICC of 0.90 (SEM, 1.40 seconds) ${ }^{[18]}$ Single hop test ${ }^{[5,19]}$ : For the single-hop test, participants 
Table 1: Mean (standard deviation) for each functional performance test

\begin{tabular}{|llcc}
\hline Groups & FAI group & FAI limb & Uninvolved limb \\
& Single-limb hopping test(s) & $5.09(0.43)$ & $3.72(0.41)$ \\
& Figure of-8-hop test(s) & $7.87(0.13)$ & $7.17(0.02)$ \\
FAI-GW Group & Side- hop test(s) & $10.59(0.22)$ & $9.08(0.52)$ \\
& Single-limb hurdle test(s) & $5.16(0.23)$ & $4.10(0.14)$ \\
& Square hop test(s) & $18.96(0.26)$ & $15.90(0.63)$ \\
& Single hop test(m) & $1.50(0.03)$ & $1.51(0.01)$ \\
& Single-limb hopping test(s) & $4.01(0.41)$ & $3.74(0.06)$ \\
FAI-NGW group & Figure of-8-hop test(s) & $7.33(0.21)$ & $7.21(0.04)$ \\
& Side- hop test(s) & $9.90(1.41)$ & $8.70(0.00)$ \\
& Single-limb hurdle test(s) & $4.53(0.34)$ & $4.35(0.27)$ \\
& Square hop test(s) & $18.20(0.01)$ & $16.30(0.01)$ \\
& Single hop test(m) & $1.50(0.03)$ & $1.51(0.06)$ \\
Non-FAI group & Single-limb hopping test(s) & $3.48(0.20)$ & $3.54(0.20)$ \\
(Matched) & Figure of-8-hop test(s) & $6.92(0.12)$ & $7.05(0.11)$ \\
& Side- hop test(s) & $8.79(0.15)$ & $8.98(0.17)$ \\
& Single-limb hurdle test(s) & $3.98(0.36)$ & $4.05(0.31)$ \\
& Square hop test(s) & $15.40(0.27)$ & $15.60(0.27)$ \\
& Single hop test(m) & $1.50(0.05)$ & $1.50(0.07)$
\end{tabular}

FAI: Functional ankle instability; FAI-GW: FAI-giving way; FAI-NGW: FAI- non-giving way; s:-seconds; m: meters

were instructed to hop forward as far as possible. The distance was recorded from the position of the toes on the starting line to the end of the jump to the nearest $0.01 \mathrm{~m}$. Trial reliability for this test was good, with an ICC of 0.96 (SEM, 4.56 seconds) ${ }^{[22]}$.

\section{Statistical Analysis:}

Data was presented as mean \pm SD. Data was analyzed using the Statistic Package of Social Sciences (SPSS) version 17.0. The mean of the 3 readings i.e. final score of each FPT was used for statistical analysis. Intra group comparison was done using the paired t-test, to compare performance differences between both limbs in the FAI and non-FAI groups. Inter group comparison was done using the independent-samples t-test, to compare performance differences between the involved limb of FAI and matched limb of non-FAI group for each functional test. Results were considered to be statistically significant at $P<0.05$.

\section{RESULTS}

In our present study, on inter group comparison of the involved leg of the FAI group and matched leg of nonFAI group, the participants in the non-FAI group required significantly less time (Table-1) to complete the tests than the participants in the FAI group for all the FPTs; single-limb hopping test $(\mathrm{t}=10.12, P<0.05)$, figure-of-8 hop test $(\mathrm{t}=11.51, P<0.05)$, side-hop test $(\mathrm{t}=24.20, P<0.05)$, single-limb hurdle test $(\mathrm{t}=11.26$, $P<0.05)$, and square hop test ( $\mathrm{t}=49.60, P<0.05)$, except for the single hop test for distance $(\mathrm{t}=0.646, P>0.05)$.

On intra group comparison similar results were observed when the involved and uninvolved leg of FAI group was compared i.e. significant performance differences in the duration of the task performed were noted for all the FPTs, single-limb hopping test $(\mathrm{t}=8.78$, $P<0.05)$, figure-of-8 hop test ( $\mathrm{t}=8.25, P<0.05)$, side-hop test $(\mathrm{t}=13.70, P<0.05)$, single-limb hurdle test $(\mathrm{t}=9.98$, $\mathrm{p}<0.05)$, and square hop test $(\mathrm{t}=25.50, P<0.05)$ except for the single hop test for distance $(\mathrm{t}=1.68, P>0.05)$.

Participants in the FAI group reported a feeling of giving way (GW) while performing the tests. Twenty one $(68 \%)$ of the participants in the FAI group reported a feeling of giving way during the single-limb hopping test, seventeen (55\%) during figure-of-8 hop test, twenty nine (93\%) while performing side-hop test, twenty four (77\%) during single-limb hurdle test, thirty (96\%) during square hop test and eight (27\%) while performing single hop test. Based on this distribution the data was reanalyzed using 3 groups i.e. athletes with FAI who reported a feeling of giving way while 


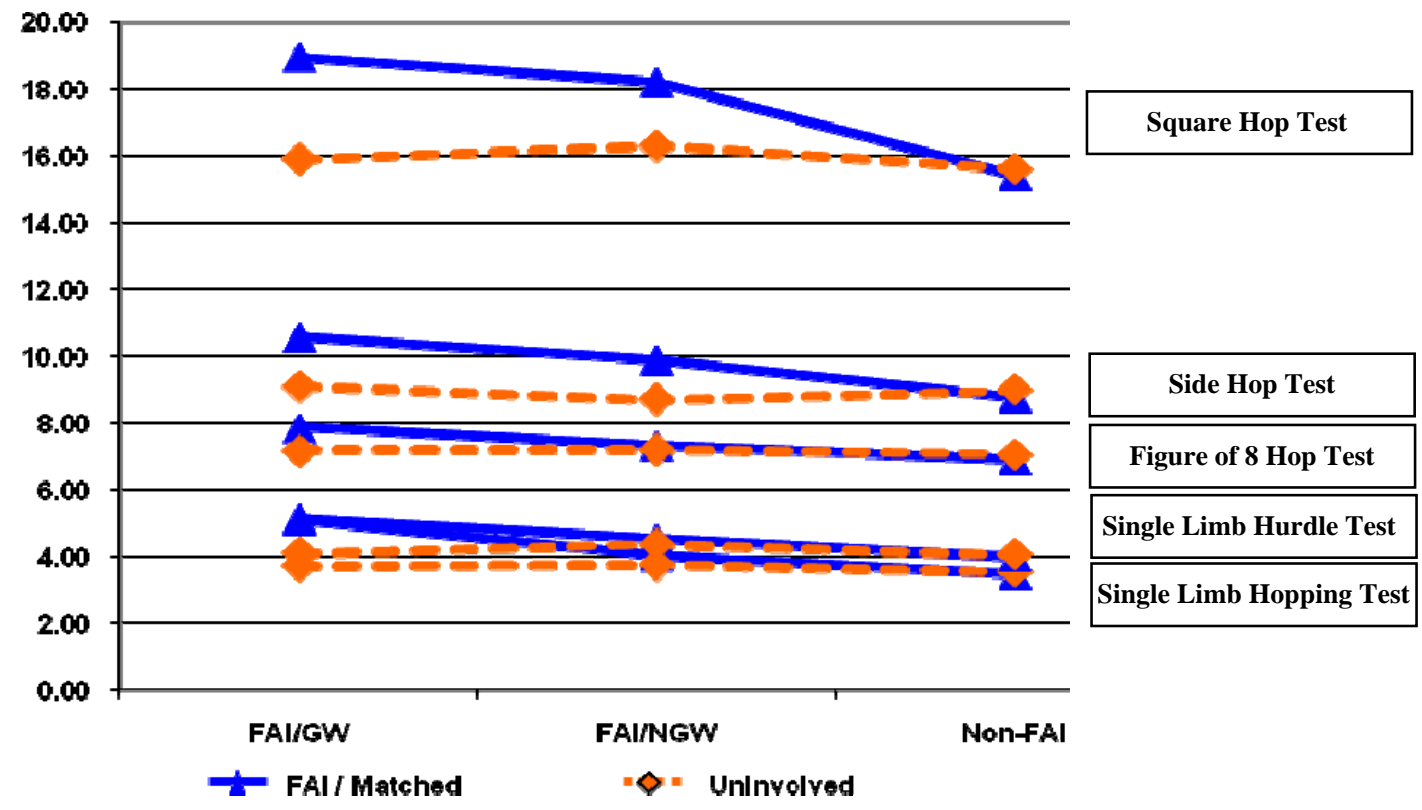

Fig. 1: Mean Time Values for the FAI / Matched and Uninvolved limb of the 3 groups FAI: functional ankle instability / GW: no giving way / NGW: no giving way

performing the tests (FAI-GW), athletes with FAI who did not report feeling of giving way while performing the tests (FAI-NGW) and the non-FAI group. For this analysis ANOVA was calculated for each functional test to determine differences amongst the FAI-GW, FAI-NGW and non-FAI groups. Since significant differences were found $(P<0.05)$, a multiple comparison Tukey (post hoc test) was calculated for any differences between the groups for the various FPTs.

Single-limb hopping test, Figure-of-8 hop test, Sidehop test and Single-limb hurdle test: Significant differences (Figure 1) in the time taken to complete the tests were observed amongst FAI-GW, FAI-NGW and non-FAI group for single limb hopping test $(\mathrm{F}=148.88$, $P<0.05)$, figure of 8 hop test $(\mathrm{F}=224.95, P<0.05)$, side hop test $(\mathrm{F}=359.18, P<0.05)$ and single limb hurdle test $(\mathrm{F}=95.92, \quad P<0.05)$. Tukey Post Hoc revealed a difference between the FAI-GW and both the FAINGW \& non-FAI group $(P<0.05)$ for all these tests. Additionally, the involved limb performed significantly worse than the contra-lateral uninvolved limb in the FAI-GW group for the single limb hopping test $(\mathrm{t}=15.07, P<0.05)$, figure of 8 hop test $(\mathrm{t}=22.86$,
$P<0.05)$, side hop test $(\mathrm{t}=14.49, P<0.05)$ and single limb hurdle test $(\mathrm{t}=16.60, P<0.05)$. No significant differences were found between the sides of the FAINGW group $(P>0.05)$ and non-FAI group $(P>0.05)$. Square hop test: Significant differences (Figure-1) in the time taken to perform the test were found between involved and uninvolved side of FAI-GW group $(\mathrm{t}=26.31, P<0.05)$. No significant differences were found between the involved and uninvolved limbs in FAI-NGW group and non-FAI group $(P>0.05)$. Thirty participants out of 31 participants in the FAI group reported a feeling of giving way for this test so Tukey Post Hoc was not performed as the FAI-NGW group had less than 2 cases.

Single hop test: No significant differences (Figure 2) in the hop distance were observed amongst any of these groups: a) FAI-GW and FAI-NGW \& non-FAI group $(\mathrm{F}=0.24, \quad P>0.05) ; \mathrm{b})$ The FAI-GW andFAI-NGW group (mean difference $=0.004, P>0.05$ ), FAI-GW and the non-FAI group (mean difference $=0.010, P>0.05$ ); c) Involved and uninvolved side of the FAI-GW group $(\mathrm{t}=1.09, P>0.05)$; $\mathrm{d})$ Involved and uninvolved limb in FAI-NGW ( $\mathrm{t}=1.35, P>0.05)$ and e) Involved and uninvolved limb in non-FAI group $(\mathrm{t}=2.09, P>0.05)$. 


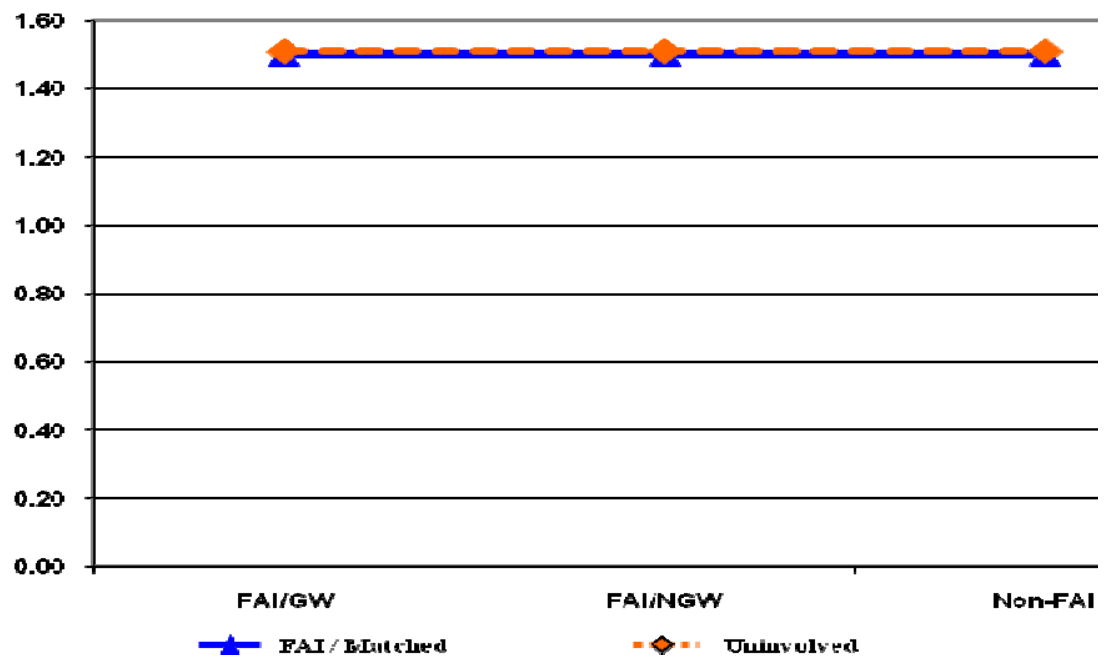

Fig. 2: Mean Distance Value for the FAI / Matched and Uninvolved limb of the 3 groups FAI: functional ankle instability / GW: no giving way / NGW: no giving way

\section{DISCUSSION}

The purpose of this study was to determine functional performance deficits in athletes with FAI compared with healthy athletes using various functional performance tests. Limited studies, evaluating the presence of functional deficits in individuals with FAI, have produced conflicting results. Only few studies have reported significant differences in performance between individuals with FAI and healthy individuals $[14,17,18]$ and some studies have reported significant differences between the involved and contra-lateral uninvolved limb of FAI individuals ${ }^{[17,18]}$. Our study observed significant performance differences between FAI and healthy individuals for all the tests involving frontal plane functional activities. The FAI group was further divided in two subgroups on the basis of presence of subjective feeling of giving way while performing the test: FAI-GW and FAI-NGW groups. The performance differences were relatively smaller between the involved leg of the FAI and the matched leg of non-FAI group when whole of the FAI group was compared; however the performance differences were particularly higher when only the FAI-GW group was compared with the non-FAI group. This feeling of giving way indicates greater performance deficits during performance of FPTs as well as actual sporting activity indicating increased severity of FAI. Significant performance differences were also observed when the involved and the contra-lateral uninvolved ankle of the FAI group were compared for the same tests.

\section{Functional performance in individuals with FAI and healthy individuals:}

In healthy individuals three joints (the talocrural joint, the subtalar joint and the tibiofibular syndesmosis) of the ankle complex work in concert to allow coordinated movement of the rear foot ${ }^{[11]}$ i.e. sagittal plane motion (plantar flexion-dorsiflexion), frontal plane motion (inversion-eversion) and transverse plane motion (internal rotation- external rotation) ${ }^{[23]}$. During functional activities stability of the ankle joints is maintained by: (1) the congruity of the articular surfaces when the joints are loaded, (2) the static ligamentous restraints, and (3) the musculotendinous units, which allow for dynamic stabilization of the joints ${ }^{[11]}$. However in individuals with ankle instability, lateral ligaments of the ankle joint (anterior talofibular ligament, calcaneofibular ligament and posterior talofibular ligament) are damaged after initial ankle sprain ${ }^{[11]}$. Due to this ligamentous damage, pathological laxity is observed in the injured joints. This may result in joint instability when the ankle is 
put in vulnerable positions during the functional activities ${ }^{[11]}$.

Functional performance and unilateral hopping tests: Single-limb hopping test: The FAI-GW group required more time to complete the test than both the FAI-NGW and non-FAI groups. Furthermore, performance differences were significantly higher for the FAI-GW group (1.4s) than the FAI-NGW group (0.3s) when the FAI limb and the contra-lateral uninvolved limb of the FAI group were compared. These findings are in accordance with the studies conducted by Jerosch et al ${ }^{[17]}$ and Buchanan et al ${ }^{[14]}$ in demonstrating that the athletes reporting the subjective feeling of giving way during the test performed worse than the athletes who did not report a feeling of giving way. The test is performed on a sloping ground surface which tests the ability of the foot to accommodate on uneven terrain by placing combined frontal and rotational stress on the ankle joint [21], consequently demonstrating performance deficits in the FAI group.

Figure-of-8 hop test and Side-hop test: Results for both these tests indicate that the FAI-GW group performed worse than FAI-NGW and non-FAI group. Performance differences in the time taken to complete the test were also observed when the FAI limb was compared to the contra-lateral uninvolved limb of the FAI group, differences being particularly higher in the FAI-GW group than the FAI-NGW group. This is in congruence with the previous studies which observed significant performance differences between the FAI and non-FAI group using this test ${ }^{[5,18]}$. However, Caffery et al ${ }^{[18]}$ did not observe any performance differences between the athletes with giving way and the athletes with no giving way and non-FAI groups for figure-of-8 hop test. It is assumed that the tests revealed the performance differences between the 3 groups as it forced the athletes to move laterally, stressing the lateral aspect of ankle joint and with that it also placed rotational stress on the ankle ${ }^{[5]}$.

Single-limb hurdle test: This test has been adapted and modified from a study conducted by Buchanan et al ${ }^{[14]}$ who failed to demonstrate any performance differences between FAI and non-FAI group using this test ${ }^{[14]}$. Our study found that the FAI-GW group required significantly more time than FAI-NGW and non-FAI group \& the difference between the FAI-GW and nonFAI group (1.18s) was much higher than between the FAI-NGW and non-FAI group (0.55s). Difference between the involved and the contra-lateral uninvolved limb was again higher for FAI-GW group (1.06s) than FAI-NGW group (0.18). These findings are consistent with literature ${ }^{[14,17,18]}$ in supporting that FAI individuals reporting instability or a feeling of giving way during tests performed worse than the individuals not reporting any instability. A possible explanation lies in the testing protocol. The height of the hurdle selected by Buchanan et al ${ }^{[14]}$ was $5 \mathrm{~cm}$ higher than height of the hurdle used in our study, which was too high for eliciting differences between the groups.

Square hop test: While performing this test significant performance differences existed between FAI and nonFAI group (3.5s). Among thirty one FAI participants thirty (96\%) reported a feeling of giving way during this test, suggesting that most of the FAI participants felt unstable while performing this test. Previous studies demonstrated performance difference between the FAI and non-FAI group using this test ${ }^{[18]}$. Our results suggest that the test can be used for discriminating between FAI and non-FAI groups. Its use in grading the severity of FAI however remains doubtful. The test required the athlete to hop in and out of a small square very quickly in multiple directions ${ }^{[18]}$, placing equally large loads on the lateral aspect of the ankle joint for both the FAI-GW and FAI-NGW groups; hence was not able to depict any significant differences between the above mentioned two groups.

Single hop test: The single hop test for distance was included in this study to elicit any performance deficits during sagittal plane functional activities in individuals with FAI. The present study observed no significant performance differences between the FAI group and non-FAI group. Also, no performance differences were identified between the limbs of FAI-GW and FAINGW groups. Previous studies also have suggested that there are no performance deficits for FAI individuals during the sagittal plane activities ${ }^{[5,19,24]}$ and greater deficits are observed in the frontal and transverse plane ${ }^{[5,24,25]}$. Our findings are consistent with these studies in stating that the sagittal plane functional activity elicits no performance difference between FAI and non-FAI individuals. This can be due 
to ankle joint instability which is caused primarily by injury to the ATFL and CFL ${ }^{[26]}$. These ligaments support the lateral aspect of ankle joint and prevent excessive inversion and rotational movement of the ankle joint ${ }^{[27]}$. The eversion-inversion movement takes place in the frontal plane and rotational movement in the transverse plane ${ }^{[23]}$ so greater deficits are observed during the FPTs which forced the ankle to move in frontal and transverse plane. However, single hop test is done primarily in the sagittal plane and thus places little stress on lateral structure of ankle joint ${ }^{[5]}$ so rendering it ineffective in eliciting performance differences between FAI and non-FAI group. Thus, clinically implying that this activity can not be used to discriminate FAI individuals and also this activity alone should not be incorporated for rehabilitation of the FAI individuals.

\section{Limb dominance and functional performance:}

Functional performance between both the limbs of the control group was compared. No significant differences were found between the limbs of the non-FAI group suggesting that limb dominance does not affect the performance in healthy individuals. These results are in congruence with findings of the previous studies ${ }^{[17,18 \text {, }}$ ${ }^{28,29]}$. As suggested by Ruiter et al ${ }^{[29]}$, the performance differences between dominant and non-dominant limb may be observed only in certain conditions like long term unilateral loading or unloading; but usually such differences are unlikely to be present. Clinical implication of this finding is that the contra-lateral limb can be used as a valid control limb, regardless whether this would be the dominant or the non-dominant limb.

This study was restricted to functional instability of the ankle; mechanical ankle instability was not considered. Future studies can consider both the aspects together to find out the exact relationship between these instabilities.

\section{CONCLUSION}

Based on the observations of the present study, it may be concluded that functional performance deficits were observed in athletes with FAI in all tests except single hop test. The deficits were specifically greater in athletes who experienced instability (FAI-GW group) during the tests. Performance differences were also identified when the involved limb was compared with the uninvolved limb of the FAI-GW group. Sagittal plane functional activities elicited no performance differences between athletes with FAI and without FAI. It was further ascertained that functional performance was not affected by limb dominance.

\section{Clinical Implication:}

These tests can be used to determine the presence of FAI and also can be used to classify the severity of disability when used along with the questionnaires for ankle instability. These tests may be incorporated in rehabilitation protocols to prevent further re-injury and can also be used as a criterion for return to play. Additionally sagittal plane activities can not be used as a criterion to identify individuals with FAI and also this activity alone should not be incorporated in rehabilitation of individuals with FAI.

\section{Future Research:}

Tests specifically stressing the lateral aspect of ankle joint should be investigated. A range of normative value in different populations, for each unilateral limb hopping test should be created for both the FAI and healthy individuals. This will help to prescreen the individuals with functional ankle instability.

\section{ACKNOWLEDGMENTS}

Gratitude is expressed to the athletes who participated in this study as well as to each of the assistants who were instrumental in the collection of the data. The researchers independently collected, analyzed, and interpreted the results and have no financial interests in the results of this study. The study protocol was approved by the Institutional Ethics Review Board, Guru Nanak Dev University.

Conflict of interests: None 


\section{REFERENCES}

1. Chan KW, Ding BC, Mroczek KJ. Acute and chronic ankle instability in the athlete. Bull NYU Hosp Jt Dis 2011;69:17-26.

2. Freeman MA, Dean MR, Hanham IW. The etiology and prevention of functional instability of the foot. J Bone Joint Surg Br1965; 47:678-85.

3. Dayakidis MK, Boudolos K. Ground reaction force data in functional ankle instability during two cutting movements. Clin Biomech (Bristol, Avon) 2006;21:405-11.

4. Demeritt KM, Shultz SJ, Docherty CL, et al. Chronic ankle instability does not affect lower extremity functional performance. $J$ Athl Train 2002;37:507-11.

5. Docherty CL, Arnold BL, Gansneder BM, et al. Functional-performance deficits in volunteers with functional ankle instability. $J$ Athl Train 2005;40:30-4.

6. Docherty CL, Gansender BM, Arnold BL, Hurwitz S. Reliability and validity of an ankle instability instrument. J Athl Train 2003;38:S-12. [abstract]

7. Hubbard TJ, Kaminski TW. Kinesthesia is not affected by functional ankle instability status. J Athl Train.2002;37:481-6.

8. Rozzi SL, Lephart SM, Sterner R, Kuligowski L. Balance training for persons with functionally unstable ankles. J Orthop Sports Phys Ther 1999;29:478-86.

9. Vaes PH, Duquet W, Casteleyn PP, Handelberg F, Opdecam P. Static and dynamic roentgenographic analysis of ankle stability in braced and nonbraced stable and functionally unstable ankles. Am J Sports Med 1998;26:692-702.

10. Hintermann B. Biomechanics of the unstable ankle joint and clinical implications. Med Sci Sports Exerc 1999;31:459-469.

11. Hertel J. Functional anatomy, pathomechanics, and pathophysiology of lateral ankle instability. J Athl Train 2002;37:364-75.

12. Cipraini JD, Haines M, O’Rand D, Levy S. Reliability and validity of a partial weight bearing measure of lower extremity performance. N Am J Sports Phys Ther 2009;4:49-59.

13. Augustsson J, Thomee R. Ability of closed and open kinetic chain tests of muscular strength to assess functional performance. Scand $J$ Med Sci Sports 2000;10:164-8

14. Buchanan AS, Docherty CL, Schrader J. Functional performance testing in participants with functional ankle instability and in a healthy non-FAI group. $J$ Athl Train 2008;43:342-6.

15. Barber SD, Noyes FR, Mangine RE, et al. Quantitative assessment of functional limitations in normal and anterior cruciate ligamentdeficient knees. Clin Orthop Relat Res 1990;255;204-14.

16. Risberg MA, Holm I, Ekeland A. Reliability of functional knee tests in normal athletes. Scand J Med Sci Sports 1995;5:24-8.

17. Jerosch J, Bischof M. Proprioceptive capabilities of the ankle in stable and unstable joints. Sports Exerc Inj.1996;2:167-71.

18. Caffrey E, Docherty CL, Schrader J, Klossner J, The Ability of 4 Single-Limb Hopping The Functional Performance Deficits in Individuals With Functional Ankle Instability J orthop Sports phys Ther 2009;39:799-806.

19. Worrell TW, Booher LD, Hench KM. Closed kinetic chain assessment following inversion ankle sprain. J Sport Rehabil 1994;3:197203.

20. Munn J, Beard D, Refshauge K, Lee RJ. Do functional-performance tests detect impairment in participants with ankle instability? $J$ Sport Rehabil 2002;11:40-50.

21. Chambers RB, Cook TM, Cowell H. Surgical reconstruction for calcaneonavicular coalition: evaluation of function and gait. $J$ Bone Joint Surg Am 1982;64:829-36.

22. Bolgla LA, Keskula DR, Reliability of lower extremity functional performance tests. J Orthop Sports Phys Ther 1997;26:138-42.

23. Huson A. Joints and movements of the foot: terminology and concepts. Acta Morphol Neerl Scand 1987;25:117-30.

24. Suda EY, Souza de RN. Functional performance assessment in individuals with ankle instability: a systemic review of the literature. Brazilian J Sports Med 2009;15:233-7.

25. Anderson KM. Movement Control and Cortical activation in Functional Ankle Instability. Available at: http://gradworks.umi.com/33/16/3316148.html. Access date: Feb, 2009.

26. Rasmussen O, Tovborg-Jensen I. Mobility of the ankle joint: recording of rotator movements in the talocrural joint in vitro with and without the lateral collateral ligaments of the ankle. Acta Orthop Scand 1982;53:155-60.

27. Stormont DM, Morrey BF, An KN, Cass JR. Stability of the loaded ankle: relation between articular restraint and primary and secondary static restraints. Am J Sports Med 1985;13:295-300.

28. LinWH, Liu YF, Hsieh CC, Lee AJ. Ankle eversion to inversion strength ratio and static balance non-FAI in the dominant and nondominant limbs of young adults. J Sci Med Sport 2009;12:42-9.

29. Ruiter de CJ, Korte de A, Schreven S, Haan de A. Leg dominancy in relation to fast isometric torque production and squat jump height. Eur J Appl Physiol 2010;108:247-55. 


\section{APPENDIX-1}

Ankle Instability Instrument ${ }^{[5,6]}$

This includes following 6 dichotomous questions (centered on the subjects' self-reported feeling of instability):

(1) Have you ever sprained your ankle?

(2) Does your ankle ever feel unstable while walking on a flat surface?

(3) Does your ankle ever feel unstable while walking on uneven ground?

(4) Does your ankle ever feel unstable during recreational or sport activity?

(5) Does your ankle ever feel unstable while going up stairs?

(6) Does your ankle ever feel unstable while going down stairs?
(Yes/No)

(Yes/No)

(Yes/No)

(Yes/No)

(Yes/No)

(Yes/No)

For each yes response a point was given and the participants who scored between 2 and 6 on the index were included in the FAI group. 